\title{
CONSTRUCCIÓN DE FACULTAD: LA ESTRUCTURA EN FUNCIÓN DEL CONOCIMIENTO Y LA RUPTURA DE TERRITORIALIDADES HACIA LA INTER Y LA TRANSDISCIPLINARIEDAD
}

\section{CONSTRUCTION OF FACULTY: THE STRUCTURE AS A FUNCTION OF KNOWLEDGE AND THE RUPTURE OF TERRITORIALITIES TOWARDS INTER AND TRANS- DISCIPLINARITY}

\section{CONSTRUÇÃO DE FACULDADE: A ESTRUTURA EM FUNÇÃO DO CONHECIMENTO E DA RUPTURA DE TERRITORIALIDADES PARA A INTER E A TRANS DISCIPLINARIDADE}

\author{
Por: RAMÍREZ GÓMEZ_Carlos Arturo
}

\begin{abstract}
Especialista en Mercadeo, Universidad de Bogotá Jorge Tadeo Lozano. Docente investigador, Administración de Empresas, Universidad De Nariño. Email: villadiegocarlos@gmail.com, Colombia.
\end{abstract}

DOI: http://dx.doi.org/10.22267/rtend.171802.78

\begin{abstract}
RESUMEN
La estructura organizacional en las universidades debe ser consistente con los Proyectos Educativos Institucionales y con la estrategia general planteada en los Planes Estratégicos de Desarrollo, en consonancia con las características de la construcción curricular de los programas, y debe facilitar el trabajo inter y trans disciplinario de docentes y estudiantes, en el marco de la ruptura de las fronteras de las relaciones de dominio territorial que obstaculizan la interacción de los departamentos académico administrativos y de los programas en una facultad. Esta propuesta plantea una alternativa en la búsqueda de este deber ser, en la medida en que el rediseño organizacional de las facultades, orientado hacia la construcción de facultad como un punto de llegada y no de partida, puede contribuir a que la facultad se convierta en una realidad y trascienda la simple división burocrática.

Para ello se estudió la universidad pública colombiana en sus características organizacionales, en el contexto mundial, se consultó a expertos y se estudiaron varios casos de rediseño organizacional, así como se acudió a la observación etnográfica.
\end{abstract}


El resultado es una propuesta de estructura de facultad flexible y dinámica que elimina las territorialidades burocráticas; además, implica un cambio funcional, normativo y de cultura organizacional.

Palabras clave: estructura, territorialidad, UDDAS, interdisciplinariedad, transdisciplinariedad y función académica.

JEL: 038, M14.

\begin{abstract}
The organizational structure in universities must be consistent with Institutional Educational Projects and with the overall strategy proposed in the Strategic Development Plans, in line with the characteristics of the construction of curricular programs, and should facilitate the job of inter-and trans disciplinary of teachers and students, in the framework of the breakdown of the boundaries of the relations of territorial domination, which hinders the interaction of departments academic and administrative programs in a faculty. This proposal presents an alternative in the search of this must be, to the extent that the organizational redesign of the faculties, oriented toward the construction of the faculty as a point of arrival and not of departure, can help the school become a reality and transcend the simple division of bureaucratic.
\end{abstract}

To this end we studied the public university in Colombia in their organizational characteristics, in the global context, we consulted with experts and studied several cases of organizational redesign, as well as went to the ethnographic participant observation.

The result is a proposal for the structure of faculty, flexible and dynamic, which eliminates the territorialities of bureaucracy; it also implies a functional change, regulatory, and organizational culture.

Keywords: structure, territoriality, UDDAS, inter-disciplinarity, trans-disciplinarity and academic role.

JEL: 038, M14.

\title{
RESUMO
}

A estrutura organizacional das universidades deve ser consistente com os Projetos Educativos Institucionais e com a estratégia geral apresentada nos Planos Estratégicos de Desenvolvimento, em consonância com as características da construção curricular dos programas, e deve facilitar o trabalho inter e multidisciplinar de docentes e alunos, no âmbito da ruptura das fronteiras das relações de domínio territorial, o que dificulta a interação dos departamentos acadêmicos e administrativos dos programas em uma faculdade. Esta proposta representa uma alternativa na busca deste dever ser, na medida em que o redesenho organizacional das faculdades, voltado para a construção de faculdade como um ponto de chegada e não de partida, 
pode contribuir para que a faculdade se torne uma realidade e transcenda a simples divisão burocrática.

Para isso, o estudou na universidade pública colombiana em suas características organizacionais, no contexto mundial, foram consultados especialistas e estudaramse vários casos de redesenho organizacional, assim como recorreu à observação etnográfica.

O resultado é uma proposta de estrutura de faculdade flexível e dinâmica, que elimina as territorialidades burocráticas; além disso, implica uma mudança funcional, normativo e de cultura organizacional.

Palavras-chave: estrutura, territorialidade, UDDAS, inter disciplinariedad, trans disciplinariedad e funçãoacadêmica.

JEL: 038, M14.

\section{INTRODUCCIÓN}

Las facultades son una ilusión burocrática, es decir, no existen simplemente porque tengan presencia en el organigrama o en los estatutos, sino que su concepción corresponde a una categoría que debe construirse para alcanzar el protagonismo académico que de ellas se espera. Tal afirmación se soporta, entre otros factores, en la carencia de proyectos educativos de facultad -PEF-, la falta de interacción de los programas en lo concerniente a la integración de las funciones misionales y la ausencia de trabajo inter y trans disciplinario entre docentes y estudiantes.

En el trasfondo de esta situación organizacional subyace la concepción desde el medioevo de la organización universitaria por facultades, en razón a las afinidades epistemológicas de las disciplinas del conocimiento, en las que se enmarcan las profesiones según los objetos de estudio, de éstas o de aquéllas. Esta concepción no responde a necesidades organizacionales estructurales consecuentes con los proyectos educativos y con los planes estratégicos de desarrollo, en tanto estos pretenden dinamizar la academia y su impacto en el entorno, mientras que la estructura permanece intacta.

Esta investigación se ha realizado en razón a una propuesta de rediseño organizacional para la universidad pública colombiana, de la que se presentan aquí los resultados para la primera fase de reestructuración, la cual se fundamenta en el fortalecimiento de las facultades como un proceso de construcción que debe partir de una nueva estructura organizacional, soportada en el conocimiento y en la ruptura de territorialidades, de tal manera que se privilegie el trabajo inter y trans disciplinario.

La segunda y tercera fase, que escapan a este artículo, proponen, paradójicamente, la desaparición de las facultades, el protagonismo de los programas académicos y finalmente la estructura matricial por proyectos y funciones. 


\section{FUNDAMENTO TEÓRICO}

Los aportes de Mintzberg en torno a la relación entre estrategia y estructura y sus tipologías de estructuras organizacionales (Mintzberg, 1979) y el diseño de organizaciones efectivas (Mintzberg, 2000), en los cuales se desarrolla el concepto de la estructura como consecuencia de la estrategia, postulado al que no atiende la universidad púbica colombiana, dado que a pesar delos cambios estratégicos la estructura novaría;la estructurapor proyectos de Daft (2000), en la que se contempla un diseño basado en proyectos productivos y no en divisiones burocráticas, precepto que no refleja una estructura por facultades; el modelo matricial de Bueno (1996) y el de Toribio (1999), en los que se desarrolla la articulación de dos dimensiones organizacionales, por ejemplo: departamentos administrativos de apoyo con unidades de negocios, que debería traducirse en las universidades, por ejemplo, entre la administración y la academia; la concepción de la organización horizontal de Ostroff (1999), en la que se reducen al máximo los niveles jerárquicos, aspecto que la universidad pública colombiana refuta en la práctica a través de su estructura altamente burocratizada; los enfoques críticos sobre la estructura, particularmente lo expuesto en el VII Seminario de Nuevo Pensamiento Administrativo, 2016, en Cali, en los que se destaca la simbología de las relaciones de dominio entre dirigentes y dirigidos, relaciones que las estructuras universitarias perpetúan, y los enfoques sobre flexibilidad en la educación superior de Díaz (2003), en los que se considera no solamente los aspectos curriculares sino los organizacionales y la correlación entre estos, interacción que las facultades no implementan, pues los cambios curriculares no trascienden a reformas organizacionales o no se realizan por limitaciones de las formas organizacionales, constituyen la esencia del marco teórico de esta investigación.

El marco normativo del Estado colombiano en materia de Educación Superior incluye el concepto de autonomía universitaria, y en ningún caso restringe la forma de organización que al interior de una facultad puede instaurarse en razón a sus características de evolución organizacional, en consonancia con los avances académicos.

En resumen, la necesidad de democratizar la organización desde la estructura, el cambio en las funciones de los docentes, el trabajo en equipo, la flexibilización organizacional administrativa y académica, la correlación estructural entre conocimiento, profesiones y unidades administrativas y la ruptura de las territorialidades generadas por departamentos burocráticos que dividen las instancias académicas, se erigen como los fundamentos teóricos y conceptuales de esta propuesta.

\section{METODOLOGÍA}

La investigación se soporta en el método etnográfico, en razón a que se considera la estructura organizacional, tanto por facultades como al interior de estas, no sólo como una manifestación racional e instrumental sino que obedece en gran medida a circunstancias históricas, a intereses políticos, a relaciones de poder entre los actores involucrados y a los temores hacia los cambios de ruptura paradigmática, aspectos que conforman condiciones comportamentales que determinan las relaciones organizacionales. De otra parte, se revisó la documentación de las universidades 
públicas y privadas, del orden regional, nacional e internacional; se consultó y se confrontó la propuesta con expertos regionales y nacionales, y se analizaron tres casos particulares de rediseño organizacional de universidades, así como la experiencia en procesos de rediseño organizacional liderados por el autor en instituciones educativas y en una universidad pública.

Esta propuesta fue ampliamente discutida en asambleas universitarias y con expertos en la Universidad de Nariño, e igualmente con directivos de la institución, en el sentido de evaluar su viabilidad para la reforma estatutaria, proceso que tardó más de un año y que finalmente arrojó como resultado la aprobación general de los estamentos universitarios, aunque actualmente continúa el debate ante el Consejo Superior para una decisión final.

Es un estudio de tipo cualitativo, puesto que no es su propósito generalizar comportamientos organizacionales en función de estadísticas y de métodos cuantitativos sino de enfatizar en tendencias cualitativas y de apoyarse en casos específicos, a través de los cuales se pueda inferir una alternativa de reestructuración que permita la construcción de facultad, en el sentido de posibilitar la interacción de sus diferentes unidades académicas y administrativas. Su alcance es del nivel propositivo.

\section{RESULTADOS}

\section{IV.1. Tendencias organizacionales de las universidades}

Una revisión del estado del arte de los estudios aplicados al diseño organizacional en las universidades evidencia la concordancia entre los autores, en aspectos vitales en los que las universidades o las instituciones de educación superior deben centrar sus esfuerzos de rediseño organizacional, en razón a las exigencias y características del ambiente global y competitivo en que se desenvuelven.

En síntesis, los autores enfatizan, de una parte, en los problemas de estructura y funcionamiento organizacional, entre los que se mencionan: Centralismo en la toma de decisiones, organización de tipo burocrático, rigidez en la estructura, divorcio entre los procesos y la estructura, ausencia de interacción adecuada entre las funciones administrativas y las misionales, subsistemas organizacionales que se obstaculizan entre sí y la carencia de sinergia entre la estructura y los sistemas de información y entre la estructura y la estrategia; y de otra parte, todos coinciden en la urgencia de que la universidad del siglo XXI dinamice su estructura organizacional para resolver estas problemáticas de cara a una educación superior que responda a las exigencias de un entorno global cada vez más competitivo, de mayor desarrollo tecnológico, con nuevas dimensiones de la responsabilidad social, para lo cual se requiere de diseños organizacionales en función del cambio permanente y de la potenciación de la relación entre los procesos administrativos y los académicos.

En efecto, en Chile (Guerrero, 2007), en Venezuela (Pertuz, 2009), en Colombia (Torres/ Padilla, 2011), en México (Enriquez, Oaxaca Perez, Olguin Romero, \& Olivares Cardenas, 2011), para citar algunos casos en Latinoamérica, estos estudios enfatizan en tales problemas 
estructurales de las formas de organización, e igualmente en Norteamérica (Hanna, 1998), (Marshall, 2010) y China (Wang \& Zhang , 2008), para mostrar otros ejemplos.

En lo referente a las propuestas de solución, en los diferentes puntos cardinales del planeta se trabaja incansablemente en nuevas perspectivas organizacionales para las universidades, las que comprenden la interacción entre múltiples aspectos institucionales. Así por ejemplo, para Pertuz (2009), quien acoge la propuesta de Campos (2002) define los subsistemas Ideológico, Orgánico-estructural, Tecnológico, Psico-social y Normativo, como los que deberían reflejarse en una estructura que los integre, aunque no propone cuál sería ésta; no obstante, este autor en el 2014 le agrega las dimensiones de Realidad Social y Realidad Económica, en el sentido de que los subsistemas definidos deben integrarse en función de estas dos dimensiones, mas no plantea una estructura organizacional en la que se plasme esta correlación. Sin embargo, en el caso del funcionamiento académico en las facultades, propone una estructura matricial, en la que interactúen los programas académicos con los departamentos de servicio (Pertuz, 2009).

El aporte de Pertuz y Campos se da en dos sentidos: de un lado, la precisión de las dimensiones anotadas, lo cual enfatiza en una correlación organizacional novedosa para las universidades, entre ámbitos no considerados antes y menos la necesidad de que se refleje en la estructura organizacional, aunque no proponen una estructura que la contenga; y de otro, aparece la urgencia de que la estructura responda a las realidades del entorno.

Guerrero identifica, en el caso chileno, (2007), la necesidad de correlacionar estructura y estrategia, aunque tampoco plantea una propuesta; Rico y Fernández (2002) introducen desde Madrid la perspectiva simbólica del diseño organizacional, pero no desemboca en una propuesta estructural; Hanna (1998), enfatiza en que en la era de la competitividad digital, las estructuras deben responder al tipo de universidad en cuestión y las clasifica en siete clases: las tradicionales, las de adultos, las a distancia, las corporaciones, las de carácter industrial con alianzas estratégicas, las de certificación de competencias y las universidades multinacionales, e igualmente recomienda varios factores de cambio en materia organizacional, pero tampoco concreta una propuesta de estructura para cada caso; en el caso de Wang y Zhang (2008), en las universidades chinas, los autores elaboran un diseño basado en la interacción de la innovación tecnológica, los procesos y los sistemas de información, pero se quedan en una departamentalización básica que no evidencia esta interacción. Se destaca la interacción entre la tecnología de soporte y los negocios, a manera de mapa de procesos, pero esto no corresponde a una estructura organizacional. El aporte de estos autores consiste en anotar la importancia de los sistemas de información en el funcionamiento de la estructura organizacional, lo cual evidentemente debe facilitar los procesos; sin embargo, el tratamiento que se le da a la propuesta no integra los departamentos de soporte con los departamentos funcionales del resto de la organización.

En Marshall (2010) hallamos el concepto de modalidades de educación y su correlación con el cambio organizacional con base en el uso de tecnologías, aunque no presenta una estructura acorde con la argumentación; para Enriquez, (Enriquez, Oaxaca Perez, Olguin Romero, \& Olivares Cardenas, 2011), en relación con convertir a las universidades 
en organizaciones que aprenden, en el contexto mexicano, clasifican los procesos en estratégicos, de soporte y operacionales y exponen la interrelación entre ellos, en función de una cadena de valor, pero no lo llevan hasta una estructura como tal, pues no basta definir el mapa de procesos sino que hay que diseñar la estructura administrativa que facilite su efectividad, y hasta allí no llegan los autores.

Finalmente, para Torres, (2013), el diseño organizacional en las universidades colombianas debe privilegiar la descentralización funcional, más no propone una estructura que realice tal propósito, lo cual refirma el carácter propositivo de esta investigación.

Igualmente, cabe destacar a Mario Díaz, quien aboga por la flexibilidad de la Educación Superior Colombiana, lo que incluye la flexibilidad administrativa (2003), es decir, estructuras que faciliten la articulación con las funciones académicas y puedan adecuarse permanentemente a las nuevas exigencias curriculares o que propicien estos cambios, esencialmente en función del conocimiento y no de requerimientos funcionales rígidos.

En resumen, los autores consultados introducen importantes conceptos, contextos y dimensiones propositivas, pero existen grandes vacíos entre sus planteamientos y las estructuras propuestas, cuando las hay, pero en la gran mayoría prevalece el concepto y la estructura de facultad.

El estudio de las propuestas y consideraciones nacionales e internacionales en torno a la estructura organizacional arroja seis tópicos de interés: la búsqueda de la flexibilidad, equilibrio entre centralización y descentralización, correlación entre estructura y responsabilidad social, estructura y democratización, estructuras matriciales y el fortalecimiento de las facultades. En este último caso, la organización por facultades sigue prevaleciendo en el mundo universitario, a nivel local, regional, nacional e internacional, y con ello un fuerte énfasis en su fortalecimiento, ya que como unidad estructural de la organización de la academia y en su supuesta función integradora de las diferentes unidades académicas y administrativas que la componen, es la unidad organizacional que en principio puede potenciar la funcionalidad entre la centralización y la descentralización, especialmente en sus órganos directivos y decisionales.

Las facultades siguen siendo la unidad fundamental en la estructura académico administrativa de las universidades, y los esfuerzos de reestructuración se centran en fortalecer su autonomía y descentralización funcional, tanto en lo académico como en lo administrativo. Este fortalecimiento se traduce no sólo en lo financiero sino en lo organizacional, en términos de dotar a las facultades de unidades académicas y administrativas de apoyo, facilitadoras de la integración de las funciones misionales; no obstante, la ausencia de propuestas estructurales consistentes con estos propósitos es el común denominador de los estudios. 


\section{IV.2. La estructura organizacional en la universidad pública colombiana}

La Universidad pública colombiana se ha estructurado organizacionalmente con base en condiciones históricas particulares que han dado pie a una estructura por facultades y a unas unidades administrativas de apoyo. Es generalizada la estructura funcional, por departamentalización. La mayoría presenta una estructura que refleja las tres funciones misionales como determinadoras de la organización. Así, la docencia, la investigación y la interacción o proyección social tienen asiento en unidades organizacionales de orden directivo, que a su vez administran las unidades encargadas de ejercer estas funciones misionales. En la mayoría de los casos estas unidades se denominan Vicerrectorías, y en otras, Direcciones, aunque la función de interacción social no se refleja en una de estas divisiones de manera autónoma, pues cuando aparece se muestra unida a la vicerrectoría de investigaciones. De otra parte, la ley obliga a la presencia de dos instancias rectoras superiores como lo son el Consejo Superior y el Consejo Académico. Existe un rector, como ejecutivo, y sus diferentes organismos asesores, más o menos similares. Y de igual forma, la función administrativa adquiere el estatus de vicerrectoría, y en mayor o menor medida aparecen unas unidades de apoyo académico.

Dentro de las facultades, aparecen los mismos organismos funcionales, como las decanaturas, el consejo de facultad, los departamentos académico administrativos, los programas académicos, los comités curriculares y de investigaciones o los de plan de estudios, y en pocos casos los centros de investigación. En otras palabras, las funciones misionales, las administrativas y las de apoyo académico, así como los organismos asesores conforman el corpus básico de la estructura organizacional de la universidad pública colombiana.

De otra parte, son estructuras altamente burocratizadas en función de un excesivo centralismo en la toma de decisiones, una alta especialización del trabajo, poco trabajo en equipo, cadenas de mando largas, tramos de control cortos, es decir, pocas personas al mando de un jefe, y una intrincada red de estatutos y normas.

En esta caracterización ha sido determinante la normatividad y las políticas oficiales que regulan la educación superior en Colombia, en relación a organismos directivos, a exigencias de control, a la agrupación por programas, departamentos y facultades incluso; no obstante, en el marco de la autonomía universitaria no se ha legislado sobre la forma interna de organización de una facultad.

\section{IV.3. Estructura y flexibilidad en la universidad pública colombiana}

En términos generales, la función administrativa sigue constriñendo las posibilidades de flexibilidad académica en las universidades. Cada que se plantea la posibilidad de renovar procesos académicos se encuentra que la rigidez administrativa lo impide, de tal forma que en muchos casos es utilizada esta situación como excusa para no generar los cambios que la academia requiere. 
La estructura administrativa se ha constituido sobre un modelo curricular asignaturista, modelo imperante en la universidad colombiana, en el cual cada docente trabaja aisladamente y se siente único dueño de la cátedra. Este determinismo de lo administrativo sobre lo académico se extiende incluso a la dificultad para integrar las funciones misionales en el quehacer académico, como lo son la docencia, la investigación y la interacción social, integración que requiere del trabajo inter y transdisciplinario, que rebasa la asignatura y que exige nuevas formas organizacionales en lo administrativo de apoyo y en la misma organización de la academia.

En esta primera fase, objeto de esta disertación, se propone una alternativa de estructura organizacional de las facultades que posibilite la construcción de las mismas y rompa las territorialidades en función del trabajo inter y trans disciplinario de docentes y estudiantes.

\section{IV.4. El problema de las territorialidades}

La estructura generalizada por facultades y dentro de estas por departamentos académico administrativos y por programas, a los cuales se adscriben los docentes, ha traído consigo la territorialidad académica y administrativa, entendiéndose ésta como el aislamiento de las unidades organizacionales y sus integrantes en términos de consolidar feudos de poder, políticos y cognitivos, que obstaculizan la sinergia entre las diferentes unidades de la facultad. Esta territorialidad ha desembocado en las siguientes situaciones que, entre otros factores, han impedido la construcción de facultad más allá de las decisiones burocráticas estructurales:

Afirma el investigador Mario Díaz en su libro Flexibilidad y Educación Superior en Colombia (2002), al referirse a este tipo de organización de estructuras rígidas, que es la que prevalece en la educación superior en Colombia, sobre los problemas que se desencadenan causados por este tipo de organización académico administrativa: Tendencia al monopolio de los profesores de cada unidad académica alrededor de un área de conocimiento específica con exclusión de aquellos que pertenecen a otras unidades; si se da la adscripción de un programa académico a la unidad académica, los profesores se sienten dueños tanto del programa de formación como de las asignaturas de dicha área; la organización alrededor de un área de conocimiento se debilita frente a la creciente relación de los profesores con el programa; amplia dedicación a las funciones de docencia con muy escasa vinculación a procesos investigativos $-y$ viceversa-, que impliquen interacción y comunicación con docentes de otras unidades. El profesor se consuma y se consume en la docencia; poca comunicación, colaboración y movilidad entre los profesores de las diferentes unidades académicas; creciente jerarquización entre docencia e investigación y, como consecuencia, creciente estratificación de los profesores entre "académicos" y "docentes"; duplicación de responsabilidades de los profesores y duplicación de los contenidos dentro y entre programas académicos; desarrollo de cursos paralelos; tendencia a la burocratización del profesorado y pérdida de la capacidad innovadora tanto de los contenidos como del contexto de aprendizaje; tendencia al aislamiento de los profesores dentro de una unidad como consecuencia del aislamiento estructural de las asignaturas de un programa académico; 
poca capacidad de autocrítica como consecuencia del sentimiento corporativista que subyace a las unidades académicas e incremento de las relaciones de poder expresadas en la jerarquización de las áreas de conocimiento de las disciplinas y entre las disciplinas. Mayor jerarquía, por ejemplo, de las ciencias naturales con respecto a las ciencias sociales.

A las cuales se le agregan estas otras (Ramírez, 2016):

Desarticulación entre disciplinas del conocimiento, unidades organizativas y profesiones; invisibilidad del trabajo disciplinar en función de la primacía del programa académico; obstaculización al fortalecimiento del trabajo disciplinario, inter disciplinario y trans disciplinario; territorialización de las relaciones de poder y confrontaciones políticas entre programas; pobre interacción entre docentes y estudiantes mediatizada más por la unidad burocrática que por el conocimiento; primacía de las relaciones de dominio burocráticas sobre las exigencias académicas de interacción entre disciplinas del conocimiento; sentido de pertenencia burocrático por parte del docente hacia su programa académico por encima de su disciplina; falta de compromiso de los docentes más allá del mínimo exigido por su programa académico; desintegración de la facultad en islas llamadas programas académicos que sólo comparten algunos créditos de formación, en detrimento de problemáticas investigativas comunes en torno a problemáticas epistemológicas y de contexto de la facultad; preponderancia de las relaciones impersonales sobre las personalizadas mediatizadas por el conocimiento y la acción interdisciplinaria; ausencia de trabajo en equipo en términos de la disciplina del conocimiento y prevalencia del trabajo individual del docente y desintegración de las funciones misionales, y alta especialización en cada una de ellas de manera aislada por parte de los profesores.

\section{IV.5. Construcción de facultad}

Es evidente la preponderancia de la reestructuración de las facultades, que desde la estructura y desde la funcionalidad allane el camino hacia la solución de estas y otras problemáticas que habrán de abordarse desde la construcción curricular de los programas académicos, como el currículo por problemas del entorno, por ejemplo, cuya repuesta organizacional pretende ser esta propuesta transitoria, en el sentido de que debe avanzarse hacia otras fases, en las que la tendencia sería la desaparición de las facultades y la visibilidad de los programas y proyectos, es decir, en la medida en que se fortalezcan las facultades en función de la interacción de las unidades académicas, estas ya no requerirían de aquellas como unidades burocráticas.

\section{IV.5.1. Contextualización temática}

La propuesta se enmarca en la descentralización, la responsabilidad social, la flexibilidad, la inter y trans disciplinariedad como búsqueda, la articulación entre disciplinas, profesiones y organización académica y administrativa, la consistencia entre estrategia, procesos, cadena de valor y estructura, la articulación de los currículos y la estructura en función de problemas epistemológicos y de problemáticas sociales del entorno, la relación entre estructura y responsabilidad social, la interacción de docentes 
mediatizada por el conocimiento y no por organismos burocráticos y la consistencia entre estructura y formación por créditos entre otros aspectos, constituyen el marco contextual temático que orienta esta propuesta de Construcción de Facultad.

\section{IV.5.2. Principios y criterios}

Ruptura de fronteras entre departamentos, programas y disciplinas. La reestructuración de facultad se orienta hacia la integración de las unidades orgánicas académicas y administrativas, de tal manera que se transciendan los límites territoriales demarcados en la actualidad por estas unidades.

Desaparecen los departamentos académico-administrativos actuales. Los departamentos actuales desaparecen y por supuesto la adscripción de los programas a estos.

Articulación entre disciplinas, profesiones y organización académica y administrativa. Se fortalece la función disciplinar en función del trabajo interdisciplinario y transdisciplinario, articulada a la profesión respectiva y a las unidades académicas y administrativas, en términos de una estructura organizacional que integre estas dimensiones.

Interacción de docentes mediatizada por el conocimiento y no por organismos burocráticos. La propuesta permite la interacción entre los docentes basada en el conocimiento y no en los obstáculos que representan las relaciones académicas determinadas por los organismos burocráticos, como lo son los departamentos.

Consistencia entre estructura y formación por créditos. La clasificación por créditos de formación en diferentes tipos, entre ellos el concepto de créditos de formación disciplinar o créditos disciplinares, exige una estructura que refleje orgánicamente los equipos disciplinares de docentes.

Interacción entre programas. Las facultades deberían integrarse por programas académicos en razón a sus afinidades epistemológicas, en torno a sus objetos de estudio, o a los objetos de transformación o a las problemáticas de contexto, etc. Estas afinidades se deben materializar en líneas de investigación, enfoques educativos, teorías curriculares, modelos pedagógicos, núcleos problémicos o temáticos, áreas de formación o en componentes de formación, entre otros, elementos estos que sin estandarizar los programas sí pueden posibilitar una mayor interacción entre estos en la medida en que puedan compartirse entre los programas académicos, es decir, en la medida en que se tornen transversales.

Adscripción de docentes y programas a la Facultad. Se contempla que los docentes pertenezcan a la Facultad orgánicamente y no a un departamento o programa específico, e igualmente los programas quedan adscritos directamente a la Facultad. 
disciplinar. Los docentes se integrarán funcionalmente en Unidades Disciplinares de Desarrollo Académico - UDDAS, conformadas por equipos de docentes con dominio en una disciplina común, quienes deben interactuar, inter y tans disciplinariamente, hacia la construcción de facultad. Cada UDDA debe tener un coordinador como función incluida en su labor académica.

Visibilización de los programas. Los programas adquieren visibilización orgánica en la estructura, ya que constituyen la unidad académica básica.

La Función académica. Se entiende la función académica como la integradora de las funciones misionales de Docencia, Investigación e Interacción Social.

El funcionario académico. Es el funcionario capaz de integrar las funciones de docencia, investigación e interacción social. Cambia, entonces el rol del docente actual.

Comité Académico por programa. Cada programa debe tener su propio comité académico el cual debe propender por la función académica.

Concepto de disciplina. Se entiende por disciplina el conocimiento esencial que representa el fundamento de una profesión y que le da identidad a ésta, y que se concreta en el conjunto de créditos de formación que sustentan un título profesional. Toda profesión tiene formación disciplinar y complementaria.

\section{IV.5.3. Descripción de la propuesta}

Es necesario precisar el concepto de facultad: La facultad es una unidad académico administrativa de la estructura organizacional de una universidad, con funciones académicas y administrativas descentralizadas, encargada de integrar las funciones de las diferentes unidades académicas y administrativas que la componen, en el marco de la articulación epistemológica de las ciencias, las artes y los saberes pertinentes, en cumplimiento de los lineamientos del PEI, y está conformada por Programas Académicos, Unidades Disciplinares de Desarrollo Académico —UDDAS, un Centro de Investigación e Interacción Social, una Escuela de Posgrados y otras unidades administrativas o académicas.

\section{IV.5.3.1. Concepto de unidad disciplinar de desarrollo académico - UDDA}

La UDDA es una unidad académica integrada por un equipo de académicos especialistas en una disciplina común, la cual es transversal a los diferentes programas de una facultad, y es la responsable de integrar las funciones misionales de docencia, investigación e interacción social en cada uno de los programas académicos de la facultad, mediante el trabajo interdisciplinario y en la medida de lo posible transdisciplinario, para lo cual se establecen las funciones estatutarias que lo permiten. Se entiende por interdisciplinariedad la conjugación de diferentes disciplinas del conocimiento en el tratamiento de problemáticas curriculares y de contexto, de tal manera que se produzca una visión holística e integral de 
éstas. Esta sinergia entre disciplinas podría dar paso eventualmente a nuevas disciplinas que adquieran características epistemológicas propias, y en este sentido se habla de transdisciplinariedad. En ambos casos es vital que existan disciplinas y personas, en este caso académicos, que en gran medida dominen el conocimiento pertinente a la disciplina que fundamenta la profesión en la que se está formando a los estudiantes, y que adquieran la cultura de trabajo en equipo con sus colegas, de tal modo que mediante un cambio estatutario de las funciones de estos académicos se propicie el trabajo entre estos equipos, espacio en el que se daría la interdisciplinariedad, con posibilidades de trans disciplinariedad, y que es la esencia de esta propuesta, en la medida en que se sostiene en el principio del dominio disciplinar como requisito para dar paso al trabajo interdisciplinar, el cual debe estatuirse contractualmente en relación a cambios normativos cuya implementación funcional vaya generando esta cultura de equipo y de interacción ente equipos.

\section{IV.5.3.2. Funciones de las UDDAS}

La misión de las UDDAS es integrar las funciones misionales de docencia, investigación e interacción social en cada programa, en la facultad y en la institución, a través del trabajo inter y trans disciplinar. Una UDDA es única en la universidad.

\section{Sus funciones son:}

Prestar los servicios de docencia en cada uno de los programas de la facultad y en los de otras facultades que lo requieran; integrar equipos interdisciplinarios con las distintas UDDAS de la facultad y de las UDDAS pertinentes de otras facultades para la docencia, la investigación y la interacción social, en función de los programas y de la facultad, en términos de problemáticas compartidas; construir interdisciplinariamente con otras UDDAS internas y externas a la facultad los currículos de cada uno de los programas de la facultad; dinamizar la evolución curricular de los diferentes programas de la facultad; participar activamente en la construcción y dinamización curricular de programas de otras facultades en las que prestan los servicios de docencia; asesorar al Consejo de Facultad y a los Comités Académicos de los programas en la formulación de directrices académicas; participar en los Consejos de Facultad ampliado a través de su coordinador; fomentar la creación de espacios transdisciplinares; formular y gestionar proyectos interdisciplinares en función de problemáticas sociales de contexto compartidas por los programas de la facultad y/o de otras facultades; consolidar las UDDAS de la facultad en función de los créditos compartidos por cada programa; operacionalizar académicamente la política y la conceptualización del trabajo académico por créditos; estructurar núcleos problémicos compartidos por los programas de la facultad en torno a problemáticas de contexto y especificidades epistemológicas, como fundamento de la construcción curricular de cada programa, en el marco de la inter y la transdisciplinariedad, y las demás que recomiende el Consejo de Facultad.

Un programa se administra y se orienta académicamente a través del director de programa, del Comité Académico y las UDDAS, entre los cuales la UDDA correspondiente a la disciplina de la profesión en la que forma el programa es la responsable no sólo del 
apoyo, staff o consultoría al programa y el respaldo en procesos de acreditación, sino de dinamizar el trabajo interdisciplinario con las demás UDDAS, dentro y fuera de la facultad, para la construcción permanente del currículo. (Ver figura 1). (En todas las figuras se ha ejemplificado con una Facultad de Ciencias Económicas y Administrativas).

Como se muestra en esta figura, la Unidad Disciplinar (UDDA) es un staff del programa, y la línea punteada significa que no hay relación jerárquica y que la UDDA pertenece a la facultad, aunque funcionalmente tiene responsabilidades con el programa y con todos los programas de la facultad, e incluso con programas externos a la facultad, tal como queda estipulado en sus funciones.

\section{Figura 1: Relación de staff de cada UDDA con el programa pertinente}

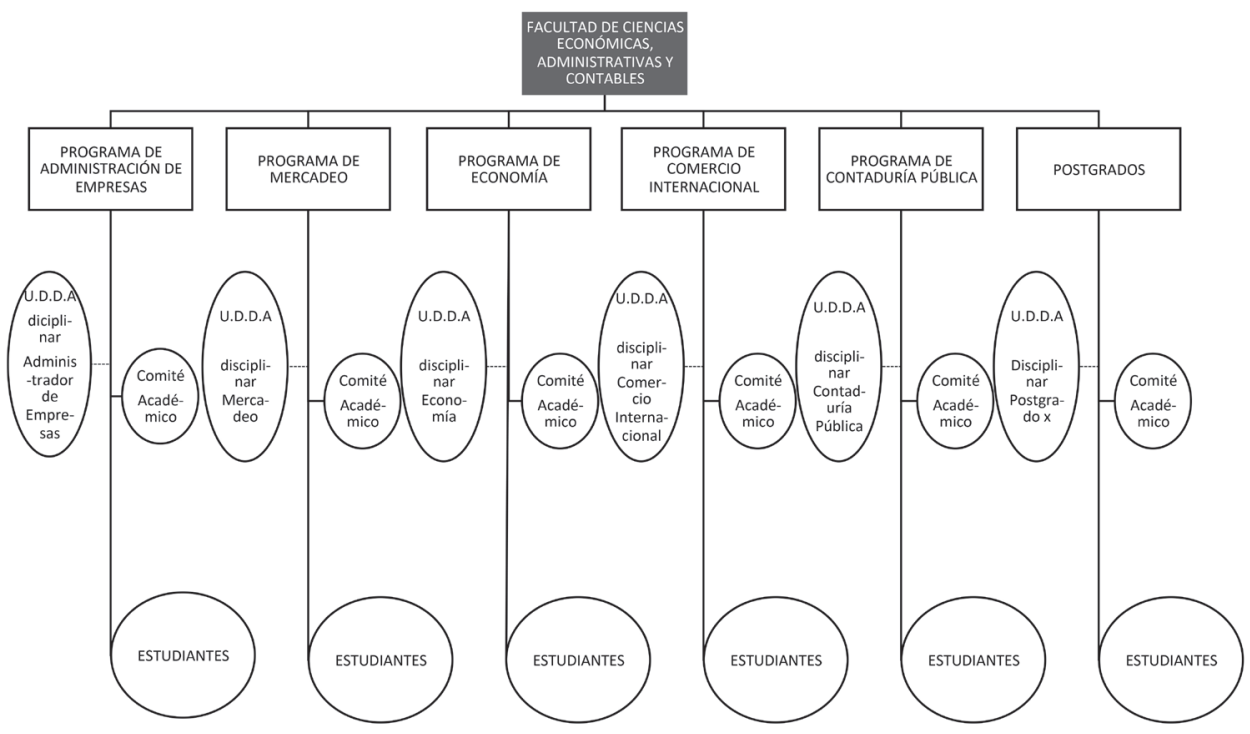

Fuente: Relación de staff de cada UDDA con el programa pertinente. (Ramírez, 2016)

En la figura 2, la línea continua entre una UDDA y su respectivo Comité Académico de su respectivo programa indica la pertinencia disciplinar del equipo con el programa, es decir, staff directo, y la línea punteada que conecta a la UDDA con los demás comités académicos y programas indica relación de staff indirecto. En otras palabras, las UDDAS tienen relación directa con su programa respectivo e indirecta con los demás programas, pero deben interactuar conjuntamente y es la UDDA respectiva a cada programa el responsable ante éste de los resultados de esta interacción.

Entre las Unidades Disciplinares de Desarrollo Académico y los programas no hay subordinación jerárquica. 
Figura 2: Estructura de facultad con las UDDAS.

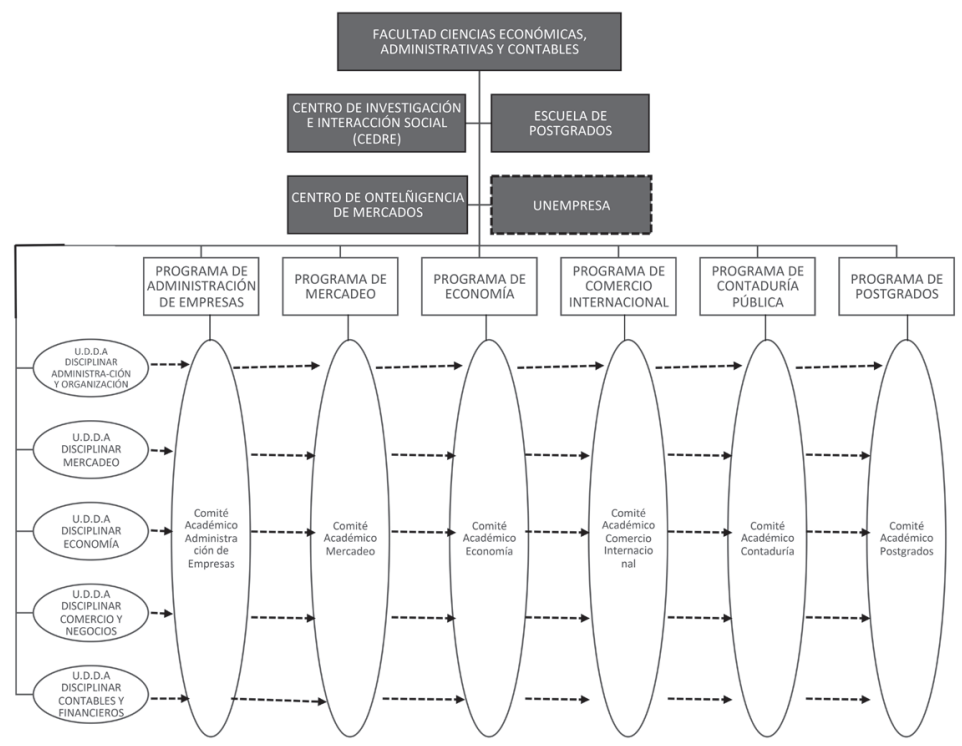

Fuente: Estructura de facultad con las UDDAS. (Ramírez, 2016)

Y en la figura 3, se representa el organigrama con línea continua para significar la responsabilidad funcional de los Equipos Disciplinares con todos los programas de la facultad, e igualmente deben interactuar con otros equipos externos a ésta.

\section{Figura 3: Estructura de facultad e interacción de las UDDAS con los Comités}

\section{Académicos}

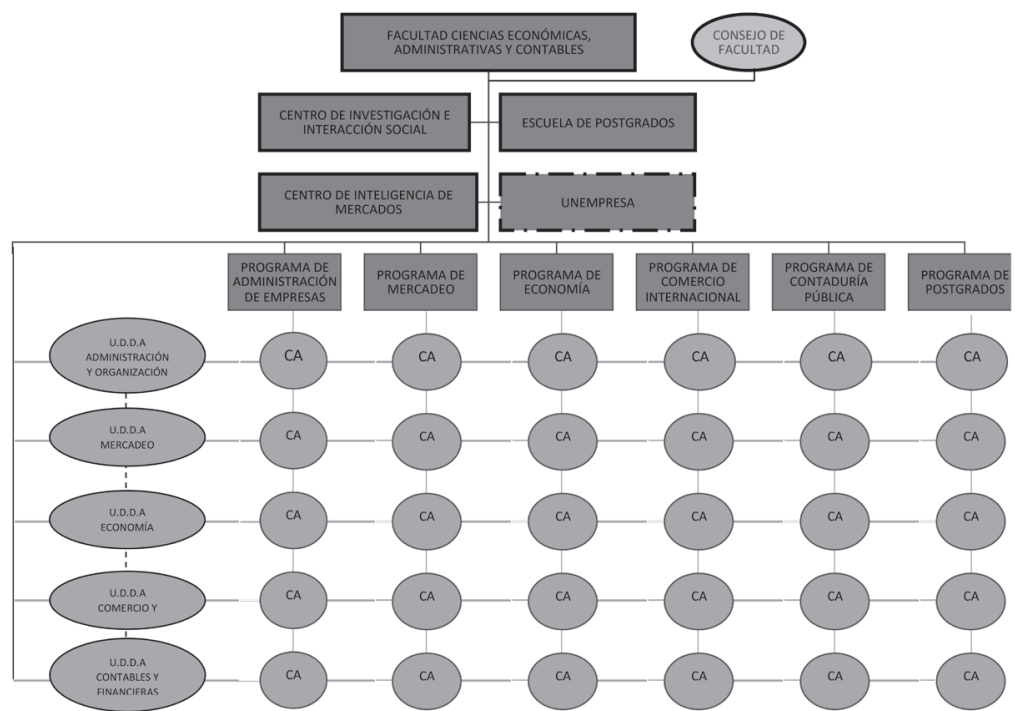

Fuente: Estructura de facultad e interacción de las UDDAS con los Comités Académicos. (Ramírez, 2016) 


\section{CONCLUSIONES Y DISCUSIÓN}

Los actuales departamentos académico administrativos que abundan en la estructura de las facultades son justamente los propiciadores de los problemas de territorialidad en éstas, al demarcar las fronteras de dominio de los docentes adscritos a ellos, así como entre los mismos departamentos, y la función de estos se circunscribe enfáticamente a administrar un programa y a prestar los servicios de docencia a otros programas. La nueva concepción, las funciones, la desaparición de los departamentos, cualquiera que sea su índole, la adscripción de docentes y programas directamente a la facultad, las funciones aquí incluidas en las UDDAS y la relación estructural y funcional, trascienden esta división territorial al romper las fronteras entre burocracia y conocimiento.

Esta concepción cuestiona seriamente la actual agrupación de programas en facultades, pues una confrontación experimental con algunas universidades ha mostrado que hay pocas facultades con programas que no comparten entre sí ninguna afinidad, al descubrir que no tienen disciplinas transversales, por ejemplo, o que el número de créditos de formación compartidos son mínimos, o que sus objetos de estudio son disímiles, o que no comparten objetos de transformación o que no se encuentran en problemáticas epistemológicas o del entorno que den pie a núcleos de formación o a líneas de investigación comunes.

La investigación ha arrojado que esta propuesta de organización de las facultades va de la mano de reformas curriculares que transformen los modelos actuales de construcción y de ejecución asignaturista, aunque es válido para los actuales modelos incluso, ya que potenciaría el trabajo disciplinar, dinamizando el interdisciplinar y generando la transdisciplinariedad, propósito último de este trabajo. No obstante, se ha presentado aquí sólo la primera fase de la propuesta, como ya se mencionó, y no las dos fases restantes, cuyas características escapan al alcance de este artículo. 


\section{REFERENCIAS}

(1) ARQUELLES, O. (2007). Una Metodologia Para El Diagnostico Y Diseño De Una Estructura Organizacional. Tesis Para Optar Por El Grado De Maestria. Mexico.

(2) BUENO. (1996). Organización De Empresas. Estructura, Procesos Y Métodos. Madrid: Pirámide.

(3) CAMPOS, B. (2002). Estrutura Y Organizacion Academico-Admnistrativa De Las Insistuciones De Educacion Superior. Coordinación General De Apoyo A La Administración De Las I.E.S. Programa Integral Para El Desarrollo De La Educación Superior (Proides) No.69, 1-6.

(4) CHANDLER, A. (1962). Strategy And Structure: Chapters In The History Of.Cambridge: The Mit Press.

(5) CLARK, B. (1992). El Sistema De Educacion Superior: Una Vision Comparativa De La Organziacion Academica.Mexico: Nueva Imagen/Uam.

(6) DAFT, R. L. (2000). Teoria Y Diseño Organizacional (6 ${ }^{\underline{a}}$ Ed.). Mexico: Thomson Internacional.

(7) DÍAZ, M. (2003). Flexibilidad En La Educación Superior Colombiana. Bogota : Men.

(8) ENRIQUEZ, N., Oaxaca Perez, G., Olguin Romero, A., \& Olivares Cardenas, M. (2011). Creatividad Y Calidad Academica: Hacia Una Nueva Estructura Organizacional Universitaria. Escuela De Graduados En Educacion, Universidad Virtual, Sistema Tecnologico De Monterrey.

(9) EVERETT, \& Rekha. (1980). La Comunicacion De Las Organizaciones. Mcgraw-Hill.

(10) GUERRERO, P. (2007). Estructura Organizacional De Las Universidades En Chile. Oikos N. 23, 733, Eae, Universidad Católica Silva Henríquez (Ucsh), Santiago De Chile.

(11) KAHN, B. K. (1996). La Psicologia Social De Las Organziaciones .New York : Wiley.

(12) MARSHALL, S. (2010). Change, Technology And Higher Education: Are Universities Capable Of Organisational Change? Wellington, New Zealand, Vol. 18, No. 3, Australasian Journal Of Educational Technology, University Teaching De. 179-192.

(13) MINTZBERG, H. (1979). Diseño De Organizaciones Eficientes. Buenos Aires: El Ateneo.

(14) MINZBERG, H. (1995). La Estructura De Las Organizaciones. Madrid : Ariel Economía.

(15) MOTOA, G. D., \& Univalle, D. D. (Noviembre De 2014). Reestructuración Univalle. (C. A. Ramirez, Entrevistador)

(16) Ostroff. (1999). The Horizontal Organization. New York: Oxford University Press.

(17) PERTUZ, B. R. (2009). Integración De Los Subsistemas De La Estructura Organizacional De Los Institutos Universitarios Venezolanos. Revista Electronica Educare, 97-117.

(18) RAMÍREZ, C. A. (2014). Rediseño Organizacional - Universidad De Nariño . Pasto.

(19) TAKEUCHI, \& Nonaka. (1999). La Organización Creadora De Conocimiento. México: Oxford.

(20) TORRES, M. R. (23 De 02 De 2013). Teorías Organizacionales Aplicadas En Las Universidades Públicas: Estudio De Caso De La Ciudad De Bogotá . Obtenido De Universidad Militar Nueva Granada: Http://Hdl. Handle.Net/10654/9965 